\title{
The liver in heart failure: a biopsy and explant series of the histopathologic and laboratory findings with a particular focus on pre-cardiac transplant evaluation
}

\author{
Christine Y Louie ${ }^{1}$, Michael X Pham², Tami J Daugherty ${ }^{3}$, Neeraja Kambham ${ }^{1}$ and \\ John PT Higgins ${ }^{1}$ \\ ${ }^{1}$ Department of Pathology, Stanford University School of Medicine, Stanford, CA, USA; ${ }^{2}$ Department of \\ Medicine (Cardiovascular Medicine), Stanford University School of Medicine, Stanford, CA, USA and \\ ${ }^{3}$ Department of Medicine (Hepatology), Stanford University School of Medicine, Stanford, CA, USA
}

\begin{abstract}
The pathologic liver changes in chronic heart failure have been characterized mostly based on autopsy series and include sinusoidal dilation and congestion progressing to pericellular fibrosis, bridging fibrosis, and ultimately to cardiac cirrhosis or sclerosis. Liver biopsies are commonly obtained as part of the work up before heart transplantation in patients with longstanding right heart failure, particularly if ascites, abnormal liver function tests or abnormal abdominal imaging are noted as part of the pre-transplant evaluation. In these cases, the liver biopsy findings may be used to further risk stratify patients for isolated heart or combined heart and liver transplantation. Thus, it is important to be able to correlate the histologic changes with post-transplant outcomes. We report the pathologic and clinical findings in liver explants from six patients who underwent combined heart-liver transplantation. We also report preoperative liver biopsy findings from 21 patients who underwent heart transplantation without simultaneous liver transplantation. We staged the changes related to chronic passive congestion as follows: stage 0-no fibrosis; stage I-pericellular fibrosis; stage II—bridging fibrosis; and stage III-regenerative nodules. Nineteen biopsies showed fibrosis with bridging fibrosis in 13 and regenerative nodules in 6 . Fifteen patients were alive at 1 year post transplant. Only three patients had a postoperative course that was characterized by signs and symptoms of chronic liver disease. Pre-transplant liver biopsies from these patients all showed at least stage II fibrosis. These patients survived for 3,6 , and 10 months after cardiac transplant. The presence of bridging fibrosis was not significantly associated with post-operative survival $(P=0.336)$ or post-operative liver failure $(P=0.257)$. We conclude that patients with bridging fibrosis may still be considered viable candidates for isolated heart transplantation. Because the pattern of fibrosis due to passive congestion is highly variable throughout the liver, a diagnosis of cirrhosis, which implies fibrosis and regenerative nodules throughout the liver, should be made with great caution on biopsy.

Modern Pathology (2015) 28, 932-943; doi:10.1038/modpathol.2015.40; published online 20 March 2015
\end{abstract}

Liver injury in the setting of heart failure results from both passive congestion due to right heart failure and ischemic injury from poor perfusion. ${ }^{1,2}$ Often, these related processes co-exist, and both contribute to liver injury. Passive congestion due to congestive heart failure may lead to pressure on the liver secondary to sustained volume overload. This

Correspondence: Dr JPT Higgins, MD, Department of Pathology, Stanford University, Room L235, 300 Pasteur Drive, Stanford, 94305 CA, USA.

E-mail: john.higgins@stanford.edu

Received 22 October 2014; revised 30 December 2014; accepted 31 December 2014; published online 20 March 2015 condition is often chronic and over time may lead to sinusoidal dilation and progressive fibrosis, changes which may be irreversible. Underperfusion of the liver may result in ischemic necrosis of the liver. This mechanism, in the absence of other damaging factors, results in coagulative necrosis of the liver parenchyma, or 'shock liver'. In contrast to chronic passive congestion, the liver may completely recover with no clinical or histologic sequelae if the patient survives the initial ischemic insult.

Defining the risk of cardiac surgery in patients with liver disease secondary to cardiac dysfunction is difficult due to the small number of studies that have investigated this subject. The risk of 
perioperative death is markedly increased by cirrhosis due to any cause,,$^{3-5}$ and cirrhosis is also associated with increased post-operative complications following cardiac surgery, ${ }^{6-9}$ including increased rates of sepsis and bleeding. Serum bilirubin has been found to correlate with operative mortality and with the presence of cardiac cirrhosis in patients with primary pulmonary hypertension undergoing heartlung transplantation. ${ }^{10}$ Few studies have examined the risk of surgery in patients with liver disease secondary to cardiac dysfunction alone, $, 8,11,12$ and there is evidence that at least a subset of patients with liver disease due to cardiac dysfunction may be able to survive cardiac transplantation with reasonable outcomes. ${ }^{8,13-15}$ Additionally, liver enzyme abnormalities and hepatic synthetic function may return to normal if cardiac function normalizes after heart transplantation. ${ }^{11,14}$

A liver biopsy may be performed in cardiac transplant candidates with heart failure in an attempt to assess the degree of liver injury related to chronic passive hepatic congestion. The findings of severe fibrosis or frank cirrhosis on liver biopsy may be used to exclude the candidate from undergoing heart transplantation with a rationale that the risk of operative mortality may be too high to justify the transplant. ${ }^{16,17} \mathrm{~A}$ small number of these patients may undergo combined heart-liver transplant ${ }^{18}$ but the remainder will likely succumb to progressive heart failure. Biopsies are often performed with a request to 'rule out cirrhosis', but chronic passive congestion does not appear to progress to cirrhosis in the same manner as chronic viral hepatitis or alcoholic hepatitis. ${ }^{2,19-23}$

The histologic changes in the setting of heart failure show a unique pattern of progression, with sinusoidal dilation preceding the appearance of fibrosis. Inflammation is minimal to absent. Pericellular fibrosis occurs first, centered on central veins, followed by central-central or central-portal bridging. Regenerative nodules are observed after the development of bridging fibrosis. At present, no staging scheme exists for the development of fibrosis in the specific context of heart disease. The existing staging systems for viral hepatitis do not directly apply as they assume a portal-based origin of fibrosis and while steatohepatitis staging schemes include pericentral fibrosis, fibrosis in chronic passive congestion shows a distinctly different pattern of evolution. ${ }^{19,21,23}$

In the current study, we report the histologic findings in explanted livers from patients receiving combined heart-liver transplants and attempt to further describe the evolution of fibrosis in patients with heart disease. We also examine the characteristics of liver biopsies from patients receiving heart transplants without concurrent liver transplant to determine whether a threshold at which patients should no longer be considered candidates for isolated heart transplantation can be established. We also propose a staging scheme for the development of fibrosis in livers showing changes secondary to chronic passive congestion from heart failure.

\section{Materials and methods}

\section{Liver Explants from Combined Heart-Liver Transplant Patients}

We identified seven cases of combined heart-liver transplants performed at our institution over a 12-year period from 2000 to 2012. One case was excluded secondary to systemic disease affecting both the heart and the liver separately (cystic fibrosis). The remaining six cases showed liver changes that could be ascribed solely to cardiac dysfunction. Additionally, no cases had any documented concurrent primary liver disease, including positive serology for viral hepatitis. For each of the six remaining cases, H\&E stained slides from formalin-fixed, paraffin-embedded material were examined; an average of six H\&E slides was available per explant case. Additional special stains, including trichrome, reticulin, and elastin van Gieson, were examined on representative sections for each liver explant. Immunohistochemical stains for cytokeratin 7, cytokeratin 19, and CD61 were also performed in each case. Pre-transplant liver biopsies were also examined when available (three of the patients). To standardize the scoring of fibrosis on liver biopsies, we staged the biopsies as follows: stage 0-no fibrosis; stage I-any degree of pericellular fibrosis short of central-central or central-portal bridging; stage II-bridging fibrosis, either central-central or central-portal; and stage III-bridging fibrosis with regenerative nodules of hepatocytes. Two of the authors (CYL and JPTH) examined each explant and also staged each biopsy. Clinical background and follow-up for each case, including age, type of heart disease, reason for transplant, and post-transplant survival, was obtained. MELD (Model for End-stage Liver Disease) scores were also calculated for each patient over 12 years of age using pre-transplant laboratory values, via the UNOS online calculator (http://optn.transplant.hrsa.gov/resources/MeldPeld Calculator.asp?index $=98$ ). MELD scores are calculated using the following formula: $0.957 \times \ln$ (creatinine $\mathrm{mg} / \mathrm{dl}$ ) $+0.378 \times \ln$ (bilirubin $\mathrm{mg} / \mathrm{dl}$ ) $+1.120 \times \ln (\mathrm{INR})+0.643$.

\section{Biopsies from Patients Receiving Heart Transplant without Concurrent Liver Transplant}

We identified 28 liver biopsies from patients at our institution who subsequently received heart transplants over a 16-year period (1997-2012). Seven cases were excluded due to concurrent causes of liver injury (chronic viral hepatitis B or C). The remaining 21 liver biopsies were examined by H\&E and trichrome stains. Histologic parameters examined included sinusoidal dilation, fibrosis (pattern 

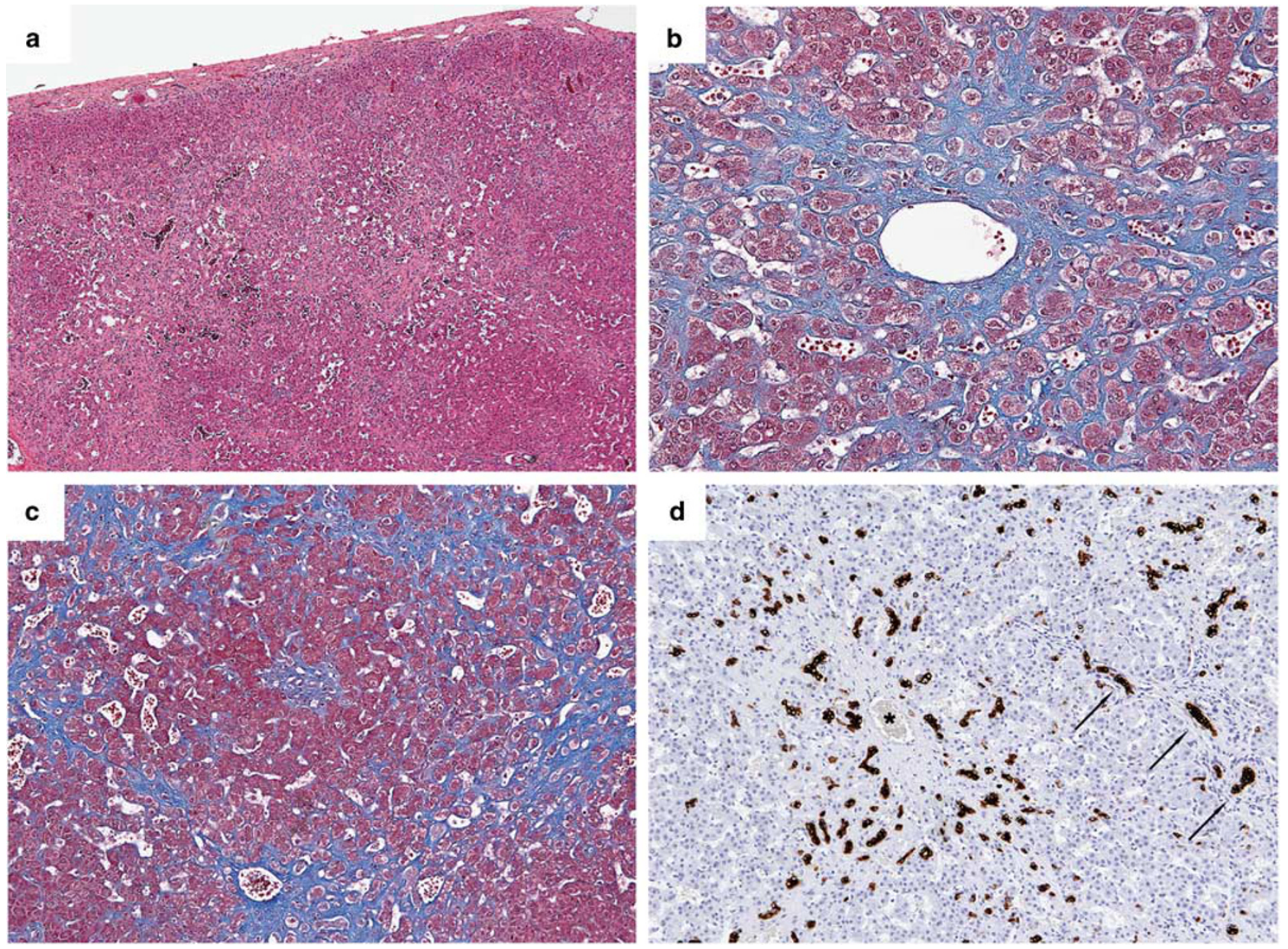

Figure 1 Histology of liver explants from combined heart-liver transplants. (a) Case V, $\times 20$ mag. Typical pattern of sinusoidal dilation and congestion, accentuated in the subcapsular area. (b) Case V, trichrome stain, $\times 200$ mag. Central vein with typical pattern of pericentral and pericellular fibrosis. (c) Case V, trichrome stain, $\times 100$ mag. Bridging fibrosis linking central veins with normal, centrally placed portal tract, so-called 'reverse polarity'. (d) Case V, ×100 mag. Cytokeratin 19 stain showing a central vein (asterisk) with biliary metaplasia of pericentral cells. A residual portal tract is at right with normal interlobular bile ducts (arrows). (e) Case III, trichrome stain, $\times 40$ mag. Subcapsular bridging fibrosis with regenerative nodules, an area suggesting cirrhosis. (f) Case III, trichrome stain, $\times 40$ mag. Another area of the same case with minimal architectural abnormality and minimal sinusoidal dilation. (g) Case IV, elastic van Gieson stain, $\times 100$ mag. Obliterated vein (probably hepatic) with evidence of re-canalization. (h) Case III, $\times 100$ mag. CD61 stain highlighting platelet fibrin thrombi in dilated sinusoids and small caliber portal vessels.

and extent), the presence of regenerative nodules, and necrosis. Fibrosis was assessed using the scoring system described in the preceding section, and each biopsy was staged by two of the authors (CYL and JPTH). Clinical background for each patient was also obtained, including pre- and post-operative laboratory values such as transaminases, alkaline phosphatase and bilirubin, MELD and MELD-XI (Model for End-stage Liver Disease eXcluding INR) scores, immediate complications post-transplant, and survival post-transplant. MELD-XI scores were calculated using the following formula: $5.11 \mathrm{x} \ln$ (bilirubin $\mathrm{mg} /$ $\mathrm{dl})+11.76 \mathrm{x} \ln ($ creatinine $\mathrm{mg} / \mathrm{dl})+9.44 .{ }^{24}$

\section{Statistical Analysis}

Comparisons between categorical variables, such as histopathology and outcomes, were analyzed for statistical significance using Fisher's exact test. For continuous variables, such as laboratory values, MELD, and MELD-XI scores, the Wilcoxon rank sum test was used. A $P$-value of $<0.05$ was considered as statistically significant. All statistical analysis was performed using $\mathrm{R}$ i386 3.1.1 for Windows (R Foundation, Vienna, Austria).

\section{Results}

\section{Liver Explants}

The six patients ranged in age from 7 to 43 years, and all received combined heart/liver transplants. One patient received a combined heart, liver, and kidney transplant. All of the patients had heart failure secondary to either congenital heart disease or idiopathic cardiomyopathy. In addition, every liver showed histologic changes that were felt to be 

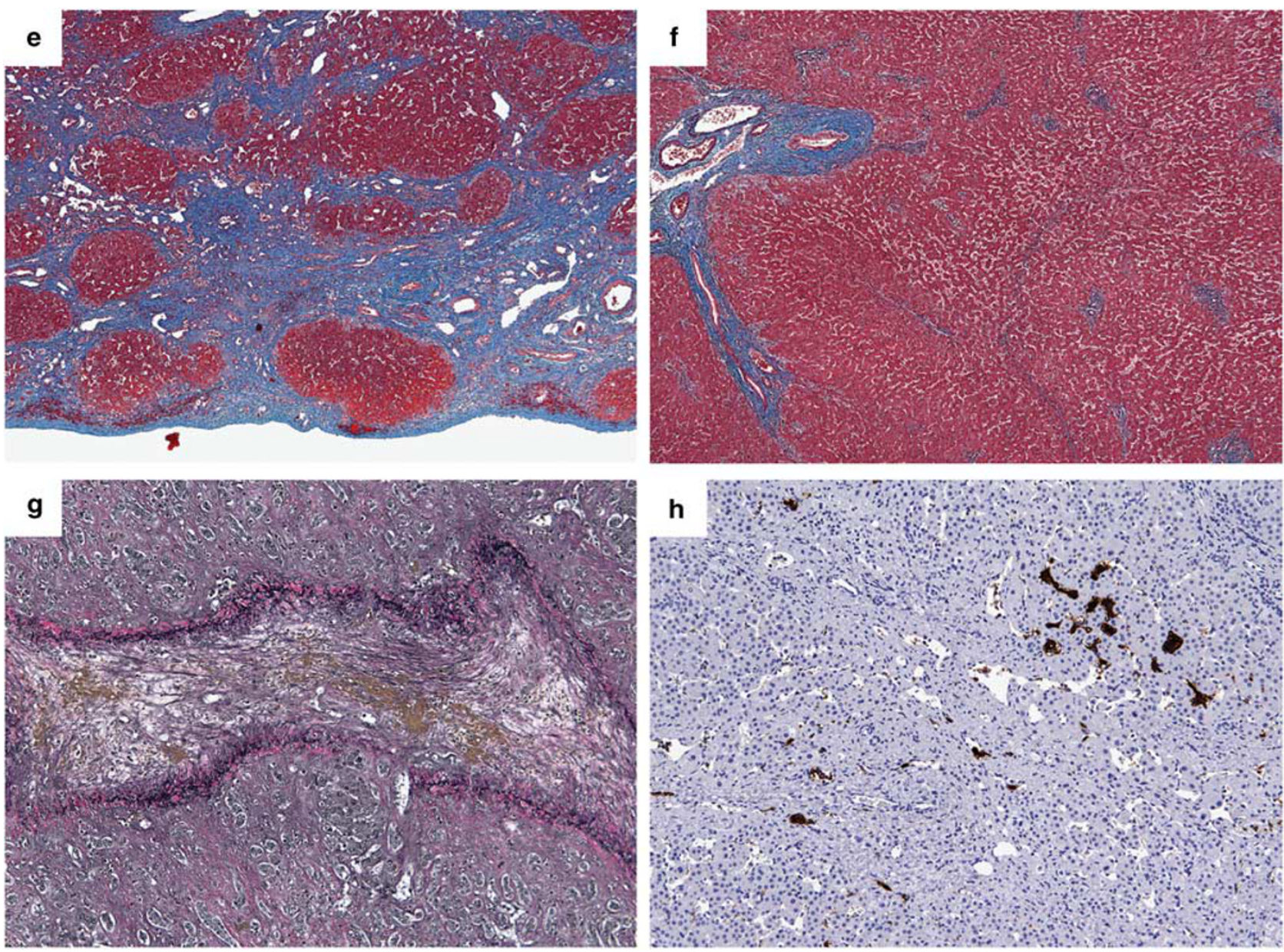

Figure 1 (Continued)

related to cardiac dysfunction alone. No changes indicative of other causes of liver injury, including viral hepatitis, were present.

Five of the six cases showed some degree of sinusoidal dilation, ranging from mild to severe. Sinusoidal dilation was usually most prominent adjacent to the capsule (Figure 1a). Trichrome stains showed bridging fibrosis in all cases and pericellular fibrosis (Figures 1b and c) in all except one case that lacked sinusoidal dilation (Table 1). The pericellular fibrosis was concentrated around the central veins. Interestingly, many cases showed a markedly patchy distribution of fibrosis. Five of the cases had areas that resembled cirrhosis. In these cases, the fibrosis appeared to be more pronounced in a subcapsular distribution (Figure 1e). Three of these cases also had areas that appeared normal (Figure 1f), often in the same block as areas that resembled cirrhosis. For example, the liver from patient III showed an especially patchy distribution of fibrosis, with some areas showing both bridging fibrosis and regenerative nodules and other areas displaying either attenuated fibrosis or appearing unaffected. The liver from patient $\mathrm{V}$ showed extensive bridging fibrosis, but mostly lacked regenerative nodules. In all of the cases, the degree of fibrosis varied markedly; three of the cases lacked areas that appeared frankly normal, but still showed some areas with much more severe fibrosis and architectural changes contrasting with areas showing only fine fibrous bridges. Reticulin stains highlighted regenerative nodules in all cases, but similar to the variable pattern of fibrosis observed, the nodules also showed a patchy distribution.

The liver architecture was further distorted by centrizonal arterioles and biliary metaplasia of cells adjacent to the central veins as recently reported by Krings et $a .^{21}$ This change was present in varying degrees in each case and was usually apparent on H\&E-stained slides. The metaplastic biliary population was particularly well highlighted by cytokeratin 19 immunohistochemistry (Figure 1d). Staining for cytokeratin 7 showed a similar appearance but also showed extensive staining of intermediate hepatocytes. Portal tracts usually lacked a ductular reaction, although in some cases the biliary metaplasia obscured the lobular architecture to such a degree that portal tracts were no longer recognizable.

We used EVG and CD61 staining to search for evidence of active or remote thrombosis (Table 1). Two cases, cases I and IV, showed obliterated veins 
Table 1 Histologic and clinical findings of six cases of combined heart/liver transplant

\begin{tabular}{|c|c|c|c|c|c|c|c|c|c|}
\hline Patient & $\begin{array}{l}\text { Age/ } \\
\text { gender }\end{array}$ & $\begin{array}{l}\text { Cardiac } \\
\text { disease }\end{array}$ & $\begin{array}{l}\text { Pre-transplant } \\
\text { biopsy fibrosis }\end{array}$ & Transplant & Explant fibrosis & $\begin{array}{l}\text { Regenerative } \\
\text { fodules }\end{array}$ & Thrombosis & $\begin{array}{l}\text { Preoperative } \\
\text { MELD }\end{array}$ & $\begin{array}{l}\text { Postoperative } \\
\text { follow-up }\end{array}$ \\
\hline I & $43 / \mathrm{F}$ & $\begin{array}{l}\text { Dilated } \\
\text { cardiomyopathy }\end{array}$ & NA & $\begin{array}{l}\text { Combined Heart/ } \\
\text { Liver }\end{array}$ & $\begin{array}{l}\text { Bridging and } \\
\text { pericellular }\end{array}$ & Yes & No & 19 & Died; 15 days \\
\hline II & $17 / F$ & Congenital & NA & $\begin{array}{l}\text { Combined Heart/ } \\
\text { Liver }\end{array}$ & Bridging only & Yes & Yes & 19 & Alive; 40 months \\
\hline III & $14 / F$ & Congenital & NA & $\begin{array}{l}\text { Combined Heart/ } \\
\text { Liver }\end{array}$ & $\begin{array}{l}\text { Bridging and } \\
\text { pericellular }\end{array}$ & Yes & No & 11 & Alive; 65 months \\
\hline IV & $30 / \mathrm{M}$ & $\begin{array}{l}\text { Dilated } \\
\text { Cardiomyopathy }\end{array}$ & III & $\begin{array}{l}\text { Combined Heart/ } \\
\text { Liver/Kidney }\end{array}$ & $\begin{array}{l}\text { Bridging and } \\
\text { pericellular }\end{array}$ & Yes & Yes & 20 & Alive; 57 months \\
\hline V & $7 / \mathrm{M}$ & Congenital & II & $\begin{array}{l}\text { Combined Heart/ } \\
\text { Liver }\end{array}$ & $\begin{array}{l}\text { Bridging and } \\
\text { pericellular }\end{array}$ & No & Minimal & NA & Alive; 39 months \\
\hline VI & $20 / \mathrm{F}$ & Congenital & II & $\begin{array}{l}\text { Combined Heart/ } \\
\text { Liver }\end{array}$ & $\begin{array}{l}\text { Bridging and } \\
\text { pericellular }\end{array}$ & Yes & No & 10 & Alive; 18 months \\
\hline
\end{tabular}

Abbreviation: NA, not applicable.

on EVG stain (Figure 1g) and a third, case II, showed a vein with eccentric intimal thickening that suggested organized thrombosis. In each of these cases, the affected vessel was located in an area of marked fibrosis. Four cases showed patchy sinusoidal and small capillary staining on CD61 stains (Figure 1h), indicating platelet-fibrin deposition. This finding was often located in the subcapsular liver, in areas with maximal fibrosis. However, the relationship of CD61 staining in sinusoids to venous thrombosis is uncertain.

Pre-transplant liver biopsies were also examined, when available (cases IV, V, and VI). The biopsies showed stage II-III fibrosis (Table 1). An additional case (case I) had a pre-transplant biopsy but the slides were not available for review; the pathology report described bridging fibrosis and architectural distortion, suggesting stage III fibrosis. The remaining two cases did not have pre-transplant liver biopsies, but one patient (case II) had severe ascites and gross findings of cirrhosis as visualized during a laparoscopic cholecystectomy, and for the second patient (case III), pre-operative imaging showed findings suspicious for cirrhosis. Pre-transplant biopsy was not performed in these cases as each patient had a clinical diagnosis of cirrhosis and it was felt that neither patient could tolerate an isolated heart transplant.

MELD scores were also calculated using pretransplant values for the patients over 12 years of age (five out of six patients). MELD scores ranged from 10 to 20, with an average of 16 . Five of the six combined transplant patients survived over a year post-transplant, with an average follow-up period of 44 months. The remaining patient died 15 days postoperatively following a repeat liver transplant 9 days after the initial combined transplant; the likely cause of the original graft failure was severe preservation injury with cardiac dysfunction also a contributing factor. The immediate cause of death was pulmonary embolism.

In summary, the histologic changes observed in the explanted livers showed a patchy distribution with much more heterogeneous involvement of the liver than is seen in end stage liver disease related to other causes. More severe fibrosis was usually noted in a subcapsular distribution. Evidence of remote thrombosis with obliterated vein lumens was noted in the areas of fibrosis in half of the cases. Whether the venous thrombosis contributed to the fibrosis and architectural changes of the surrounding parenchyma or was itself a result of the fibrosis could not be determined. Coagulative hepatocellular necrosis was not identified in any of the explant livers examined.

\section{Liver Biopsies}

Twenty-one pre-operative liver biopsies were examined in patients awaiting isolated heart transplantation. All biopsies for which collection data were available were performed via a transjugular approach (Table 3). Causes of heart disease included congenital heart disease (6), dilated cardiomyopathy (4), ischemic cardiomyopathy (3), primary pulmonary hypertension (2), restrictive cardiomyopathy (2), arrhythmogenic right ventricular cardiomyopathy (1), adriamycin-induced cardiomyopathy (1), hypertrophic cardiomyopathy (1), and senile/transthyretin type amyloidosis (1) (Table 2). Two patients received combined heart-lung transplants, two received combined heart-kidney transplants, and one received a combined heart-lung-kidney transplant. The interval between the initial liver biopsy and the subsequent transplant ranged from 10 to 579 days, with an average of 116 days.

The majority of patients were noted to have clinical ascites before transplant, and many patients additionally had abnormal liver chemistries and measures of synthetic function pre-operatively (Table 2). Eleven patients had elevated total bilirubin (based on upper limit of normal: $1.4 \mathrm{mg} / \mathrm{dl}$ ) and this was greater than $2.0 \mathrm{mg} / \mathrm{dl}$ in six patients. Each of the six patients had evidence of hepatic synthetic dysfunction based on serum albumin less than 
Table 2 Pre-transplant characteristics and laboratory values for patients undergoing heart transplant

\begin{tabular}{|c|c|c|c|c|c|c|c|c|c|c|c|}
\hline $\begin{array}{l}\text { Patient } \\
\text { number }\end{array}$ & $\begin{array}{l}\text { Age/ } \\
\text { Gender }\end{array}$ & Heart disease & $\begin{array}{l}\text { Clinical } \\
\text { Ascites }\end{array}$ & $\begin{array}{c}A P \\
(I U / L)\end{array}$ & $\begin{array}{l}\text { Total Bilirubin } \\
\quad(\mathrm{mg} / \mathrm{dl})\end{array}$ & $\begin{array}{c}A S T \\
(I U / L)\end{array}$ & $\begin{array}{c}A L T \\
(I U / L)\end{array}$ & $\underset{(\mathrm{g} / \mathrm{dl})}{\operatorname{Albumin}}$ & $\begin{array}{l}\text { Total protein } \\
\text { (g/dl) }\end{array}$ & \multicolumn{2}{|c|}{ INR MELD } \\
\hline 1 & $53 / F$ & Ischemic & Yes & 322 & 3.0 & 20 & N/A & 3.7 & 7.2 & 1.4 & 23 \\
\hline 2 & $48 / \mathrm{M}$ & $\begin{array}{l}\text { Arrhythmogenic RV } \\
\text { cardiomyopathy }\end{array}$ & Yes & 126 & 1.3 & 26 & 20 & 3.5 & N/A & 1.3 & 14 \\
\hline 3 & $53 / \mathrm{M}$ & Dilated cardiomyopathy & No & 271 & 0.5 & 26 & 20 & 2.1 & 4.9 & 1.3 & 16 \\
\hline 4 & $61 / \mathrm{M}$ & Ischemic & Yes & 60 & 4.3 & 124 & 33 & 3.4 & 6.5 & 1.6 & 24 \\
\hline 5 & $66 / \mathrm{M}$ & Dilated cardiomyopathy & Yes & 59 & 1.6 & 22 & 12 & 3.7 & 7.0 & 2.0 & 18 \\
\hline 6 & $50 / \mathrm{M}$ & $\begin{array}{l}\text { Hypertrophic } \\
\text { cardiomyopathy }\end{array}$ & Yes & 158 & 0.7 & 18 & 14 & 2.0 & 5.3 & 1.1 & 13 \\
\hline 7 & $63 / \mathrm{M}$ & Ischemic & Yes & 199 & 1.2 & 60 & 43 & 3.9 & 8.3 & 1.3 & 16 \\
\hline 8 & $60 / \mathrm{M}$ & Amyloidosis & Yes & 396 & 2.1 & 36 & 37 & 3.7 & 8.0 & 1.3 & 18 \\
\hline 9 & $40 / \mathrm{M}$ & Dilated cardiomyopathy & Yes & 184 & 1.5 & 34 & 55 & 2.3 & 6.1 & 1.6 & 15 \\
\hline 10 & $55 / F$ & $\begin{array}{l}\text { Primary pulmonary } \\
\text { hypertension }\end{array}$ & Yes & 402 & 0.5 & 24 & 50 & 3.5 & 9.6 & 1.2 & 12 \\
\hline 11 & $43 / F$ & $\begin{array}{l}\text { Adriamycin } \\
\text { cardiomyopathy }\end{array}$ & Yes & 228 & 1.8 & 22 & 24 & 2.6 & 8.1 & 1.5 & 22 \\
\hline 12 & $42 / F$ & Congenital heart disease & Yes & 311 & 1.9 & 18 & 31 & 3.6 & 7.7 & 1.9 & 27 \\
\hline 13 & $57 / F$ & Dilated cardiomyopathy & Yes & 82 & 1.7 & 42 & 82 & 3.2 & 5.7 & 1.6 & 21 \\
\hline 14 & $36 / \mathrm{F}$ & Congenital heart disease & Yes & 143 & 5.3 & 49 & 47 & 1.8 & 5.6 & 1.4 & 17 \\
\hline 15 & $36 / F$ & $\begin{array}{l}\text { Restrictive } \\
\text { cardiomyopathy }\end{array}$ & Yes & 173 & 1.3 & 35 & 30 & 4.4 & 8.5 & 1.3 & 10 \\
\hline 16 & $36 / \mathrm{M}$ & Congenital heart disease & No & 90 & 0.5 & 32 & 50 & 3.7 & 7.9 & 2.5 & 23 \\
\hline 17 & $37 / \mathrm{F}$ & Congenital heart disease & No & 61 & 2.7 & 77 & 36 & 2.3 & 4.4 & 1.5 & 20 \\
\hline 18 & 15/M & Congenital heart disease & Unknown & 118 & 0.7 & 19 & 22 & 3.0 & 7.1 & 1.5 & 11 \\
\hline 19 & $51 / \mathrm{M}$ & $\begin{array}{l}\text { Primary pulmonary } \\
\text { hypertension }\end{array}$ & No & 108 & 0.5 & 10 & 25 & 2.4 & 5.4 & 1.1 & 13 \\
\hline 20 & $49 / \mathrm{M}$ & Congenital heart disease & Yes & 1539 & 3.3 & 64 & 26 & 2.0 & 7.0 & 1.2 & 19 \\
\hline 21 & $34 / \mathrm{M}$ & $\begin{array}{l}\text { Restrictive } \\
\text { cardiomyopathy }\end{array}$ & Yes & 291 & 0.8 & 35 & 34 & 3.3 & 7.0 & 2.8 & 18 \\
\hline
\end{tabular}

Abbreviations: ALT, alanine aminotransferase; AP, alkaline phosphatase; AST, aspartate aminotransferase; INR, international normalized ratio; MELD, model for end-stage liver disease; RV, right ventricle.

$3.5 \mathrm{~g} / \mathrm{dl}$ and/or INR greater than 1.1. However, some patients were receiving anticoagulation with warfarin. For example, patient numbers 13, 16, and 21 were taking warfarin at the time of transplant, rendering their high INRs invalid as markers for hepatic synthetic dysfunction. Although alkaline phosphatase was elevated in 13 patients (based on upper limit of normal: $130 \mathrm{IU} / \mathrm{l}$ ), elevated transaminases were much less common. MELD and MELD-XI scores were also calculated for each patient using pre-operative laboratory values. MELD scores ranged from 10 to 27, with an average score of 18. MELD-XI scores ranged from 5 to 26 with an average of 17 .

With regard to histology, the majority of the liver biopsies examined showed sinusoidal dilation and congestion (Table 3; Figures 2a and c). Regenerative nodules were seen in six of the cases. None of the biopsies showed coagulative liver cell necrosis. In all, 19 out of the 21 biopsies showed increased fibrosis, in either a bridging or pericellular distribution, or both (Figures $2 \mathrm{~b}$ and $\mathrm{d}-\mathrm{h}$ ). Out of the 19 biopsies with fibrosis, 13 showed bridging fibrosis (stages II and III). From these 13 cases, 12 patients survived at least 1 month from the time of transplant. Only one out of thirteen patients showing bridging fibrosis died in the perioperative period (19 days post transplant); this death was related to right ventricular failure and pulmonary hypertension, and occurred in a 15-year-old patient with complex congenital heart disease (patient \#18). The presence of bridging fibrosis was not significantly associated with survival at 1 year post transplant $(P=0.336)$. Nine of the thirteen patients (including the one patient who died in the perioperative period) had a post-transplant course that included sepsis or excessive bleeding. The immediate preoperative bilirubin levels for these patients ranged from 0.5 to $5.3 \mathrm{mg} / \mathrm{dl}$ (average $1.9 \mathrm{mg} / \mathrm{dl}$ ).

Three of the patients (\#6, \#20, and \#21) whose biopsies showed stage II or III fibrosis showed prolonged liver failure postoperatively. All three patients died within 12 months following heart transplant. In these patients, post-transplant liver failure was characterized by persistent ascites, encephalopathy, or poor synthetic function with normal cardiac function. Patient \#6 had a preoperative total bilirubin level of 0.7 , and a 6 -month level of $1.8 \mathrm{mg} / \mathrm{dl}$. This patient's preoperative MELD score was 13. The cause of death was fungal and mycobacterial infection at 7 months after transplant. Patient \#20 had a preoperative total bilirubin level of 3.3 with a 6 -month level of $0.6 \mathrm{mg} / \mathrm{dl}$, and a preoperative MELD score of 19. This patient's death at 10 months postoperatively was related to aspiration pneumonia secondary to hepatic encephalopathy, with a total bilirubin level at 10 months of $3.0 \mathrm{mg} / \mathrm{dl}$. Both of these patients also had ascites at 6 months post-operatively, and required paracentesis 
Table 3 Histologic features of pre-transplant liver biopsies from patients undergoing heart transplant

\begin{tabular}{|c|c|c|c|c|c|c|c|c|c|}
\hline $\begin{array}{l}\text { Patient } \\
\text { number }\end{array}$ & $\begin{array}{l}\text { Biopsy } \\
\text { type }\end{array}$ & $\begin{array}{l}\text { Sinusoidal } \\
\text { dilation }\end{array}$ & Fibrosis & $\begin{array}{l}\text { Bridging } \\
\text { fibrosis }\end{array}$ & $\begin{array}{l}\text { Regene- } \\
\text { rative } \\
\text { nodules }\end{array}$ & Stage & Follow-up & $\begin{array}{l}\text { Cause of } \\
\text { death }\end{array}$ & $\begin{array}{c}\text { Prolonged } \\
\text { liver } \\
\text { failure }\end{array}$ \\
\hline 1 & TJL & Yes & Yes & No & No & I & Died; 69 months & $\begin{array}{l}\text { Allograft } \\
\text { vasculopathy }\end{array}$ & No \\
\hline 2 & TJL & Yes & No & No & No & 0 & Alive; 137 months & NA & No \\
\hline 3 & TJL & Yes & Yes & Yes & No & II & Died; 75 months & Allograft failure & No \\
\hline 4 & TJL & Yes & Yes & Yes & Yes & III & Died; 77 months & $\begin{array}{l}\text { Allograft } \\
\text { vasculopathy }\end{array}$ & No \\
\hline 5 & Unknown & Yes & Yes & Yes & Yes & III & Alive; 94 months & NA & No \\
\hline 6 & TJL & No & Yes & Yes & Yes & III & Died; 6 months & Liver failure & Yes \\
\hline 7 & TJL & Yes & Yes & No & No & $\mathrm{I}$ & Alive; 69 months & NA & No \\
\hline 8 & TJL & Yes & Yes & Yes & No & II & Died; POD \#70 & $\begin{array}{l}\text { Enterobacter } \\
\text { pneumonia }\end{array}$ & No \\
\hline 9 & TJL & Yes & Yes & No & No & I & Alive; 50 months & NA & No \\
\hline 10 & TJL & Yes & Yes & No & No & $\mathrm{I}$ & Alive; 46 months & NA & No \\
\hline 11 & TJL & Yes & Yes & No & No & I & Alive; 48 months & NA & No \\
\hline 12 & TJL & Yes & Yes & Yes & No & II & Alive; 47 months & NA & No \\
\hline 13 & TJL & Yes & Yes & Yes & No & II & Alive; 46 months & NA & No \\
\hline 14 & TJL & Yes & Yes & Yes & No & II & Alive; 43 months & NA & No \\
\hline 15 & TJL & Yes & Yes & Yes & Yes & III & Alive; 27 months & NA & No \\
\hline 16 & TJL & No & No & No & No & 0 & Died; POD\#35 & $\begin{array}{l}\text { Diffuse alveolar } \\
\text { damage }\end{array}$ & No \\
\hline 17 & TJL & Yes & Yes & No & No & I & Alive; 26 months & NA & No \\
\hline 18 & TJL & Yes & Yes & Yes & Yes & III & Died; POD\#19 & $\begin{array}{l}\text { Aspergillus } \\
\text { pneumonia }\end{array}$ & No \\
\hline 19 & TJL & Yes & Yes & Yes & No & II & Alive; 17 months & NA & No \\
\hline 20 & TJL & Yes & Yes & Yes & No & II & Died; 10 months & Liver failure & Yes \\
\hline 21 & TJL & Yes & Yes & Yes & Yes & III & Died; 3 months & Sepsis & Yes \\
\hline
\end{tabular}

Abbreviations: NA, not applicable; POD, post-operative day; TJL, transjugular liver biopsy.

during the perimortem period. The third patient (\#21) had a complicated post-operative course including status epilepticus and acute shock liver with persistently elevated transaminases and bilirubin values; this patient died 3 months post transplant from sepsis. This patient's preoperative MELD score was 18. The patient's total bilirubin level ranged between 4.1 and 41.8 post-operatively. Overall, the presence of bridging fibrosis on preoperative liver biopsy was not significantly associated with prolonged liver failure $(P=0.257)$. Also, MELD and MELD-XI scores were not found to be significantly associated with the degree of fibrosis present $(P=0.615$ and 0.228 , respectively). The patients with pre-transplant MELD scores >20 (patients \#1, 4, 11, 12, 13, and 16) were either still alive at follow-up or died of causes unrelated to liver failure.

At 6 months after heart transplantation, laboratory values were available for 17 of the 21 patients (Table 4). Only two patients showed an elevated bilirubin level, at levels of 1.8 and $1.9 \mathrm{mg} / \mathrm{dl}$ (patients \#6 and \#19, respectively). Four patients had an elevated AST level (based on upper limit of normal: $39 \mathrm{IU} / \mathrm{l}$ ) and five patients had an elevated ALT level (based on upper limit of normal: $59 \mathrm{IU} / \mathrm{l}$ ). INR levels, when available, averaged 1.2. Serum albumin levels averaged $3.4 \mathrm{~g} / \mathrm{dl}$. Ten patients showed elevated alkaline phosphatase levels, with an average level of $235 \mathrm{IU} / \mathrm{l}$.
At a 12-month follow-up interval, 15 patients had laboratory values available (Table 5); only one had an elevated total bilirubin level $(3.0 \mathrm{mg} / \mathrm{dl}$, patient \#20, reflecting prolonged liver failure post-operatively). Three patients had an elevated AST level (with a maximum AST of $61 \mathrm{IU} / \mathrm{L}$ ) and four patients had an elevated ALT level (with a maximum of ALT of $97 \mathrm{IU} / \mathrm{L})$. Only two patients had an INR value of greater than 1.1. Alkaline phosphatase values averaged $219 \mathrm{IU} / \mathrm{L}$, with 13 patients showing an elevated level.

One patient (\#3) underwent liver biopsy posttransplant; this patient showed stage II fibrosis (bridging, without regenerative nodules) on pretransplant liver biopsy, and the follow-up biopsy was performed 6 years post transplant for work-up for repeat heart transplantation. The repeat biopsy showed decreased fibrosis with minimal pericellular fibrosis noted, without any bridging seen on a trichrome stain (stage I).

\section{Discussion}

Studies of liver pathology in the setting of heart failure have, to date, been predominantly autopsy studies. ${ }^{19,23,25,26}$ Our report is one of the first to examine the findings in liver explants from patients receiving combined heart-liver transplants. The variable distribution of fibrosis that we noted in the liver explants has implications for pre-operative 

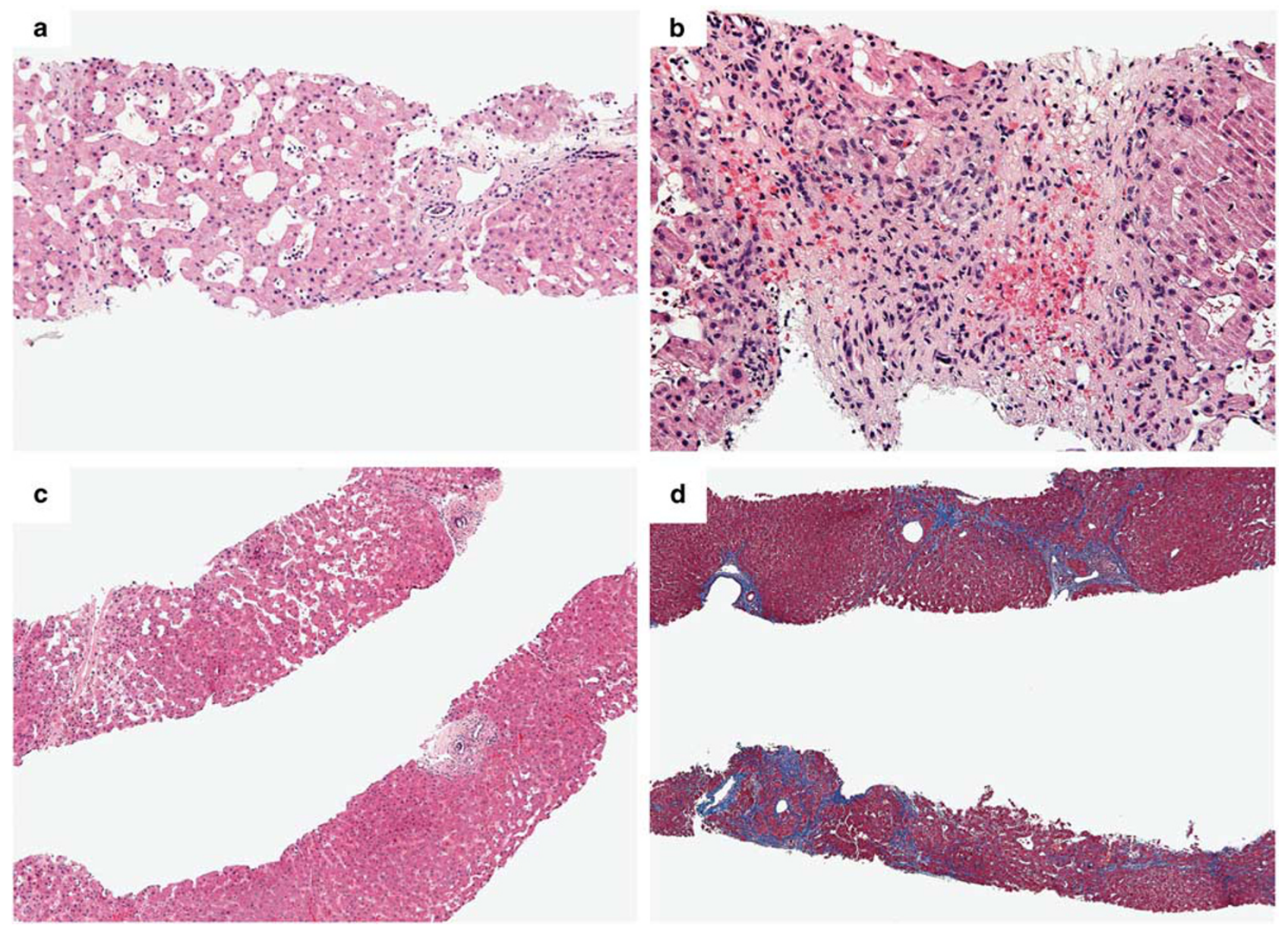

Figure 2 Histology of pre-heart transplant liver biopsies. (a) Case 18, ×100 mag. Normal portal tract with marked sinusoidal dilation in the adjacent lobule. This patient died on post-op day \#19 from fungal pneumonia. (b) Case 5, ×200 mag. Pericentral area with drop-out, fibrosis, and hemorrhage. This patient remains alive after 94 months. (c) Case 8, × 100 mag. The degree of sinusoidal dilation may vary from core to core. The upper core shows pronounced sinusoidal dilation whereas the lower shows minimal changes. This patient died on post-op day \#70 from bacterial pneumonia. (d) Case 3, trichrome, $\times 40$ mag. Bridging and pericellular patterns of fibrosis. The portal tract on the left in the upper core appears nearly normal whereas the one on the right shows several fibrous septa with bridging and nodularity in the surrounding liver parenchyma. This patient died 75 months post transplant of allograft failure. (e) Case 4 , × 40 mag. Extensive bridging and pericellular fibrosis throughout the lower core. The lobular architecture is essentially unrecognizable although there is minimal nodular regeneration. This patient died 77 months post transplant of allograft vasculopathy. (f) Case $6, \times 40$ mag. Pericellular and bridging fibrosis. This patient died 6 months post transplant of liver failure. (g) Case 8, trichrome, $\times 100$ mag. Relatively normal portal tract (left) with central hepatocellular drop-out and fibrosis with ceroid pigment laden macrophages (lower right). (h) Case 15, trichrome, $\times 100$ mag. This case displays the most advanced fibrosis seen on biopsy (stage III). This patient developed a subdural hematoma, but did well and remains alive at 27 months follow-up.

pathologic assessment of the liver as part of the work-up for either isolated heart or combined heart and liver transplantation. All but one of these explants showed areas of bridging fibrosis alternating with other areas showing preserved architecture. In each of these cases, we were able to identify areas of liver parenchyma that on a biopsy sample would have shown normal or nearly normal lobular architecture with little to no fibrosis. Therefore, obtaining multiple biopsy cores from different areas of the liver might be expected to increase the accuracy of the assessment. Sampling may be further optimized by ultrasound guidance with preferential sampling of areas that appear abnormal based on imaging. Other imaging modalities including transient elastography may also help to obtain an accurate assessment of the entire liver; however, to date, there are no studies addressing whether elastography results correlate with liver biopsy results in the context of chronic passive congestion. Elastography data were not available for any of our patients.

It is important that pathologists bear the potential variability of the distribution of fibrosis in mind when they assess liver biopsies for chronic passive congestion. One should not assume that findings on a given liver biopsy are necessarily reflective of the amount of injury present in other unsampled regions of the liver, particularly on a scant biopsy. Furthermore, the transjugular approach to the liver biopsy that is typically used in these patients precludes sampling of the most severely affected subcapsular regions of the liver. 

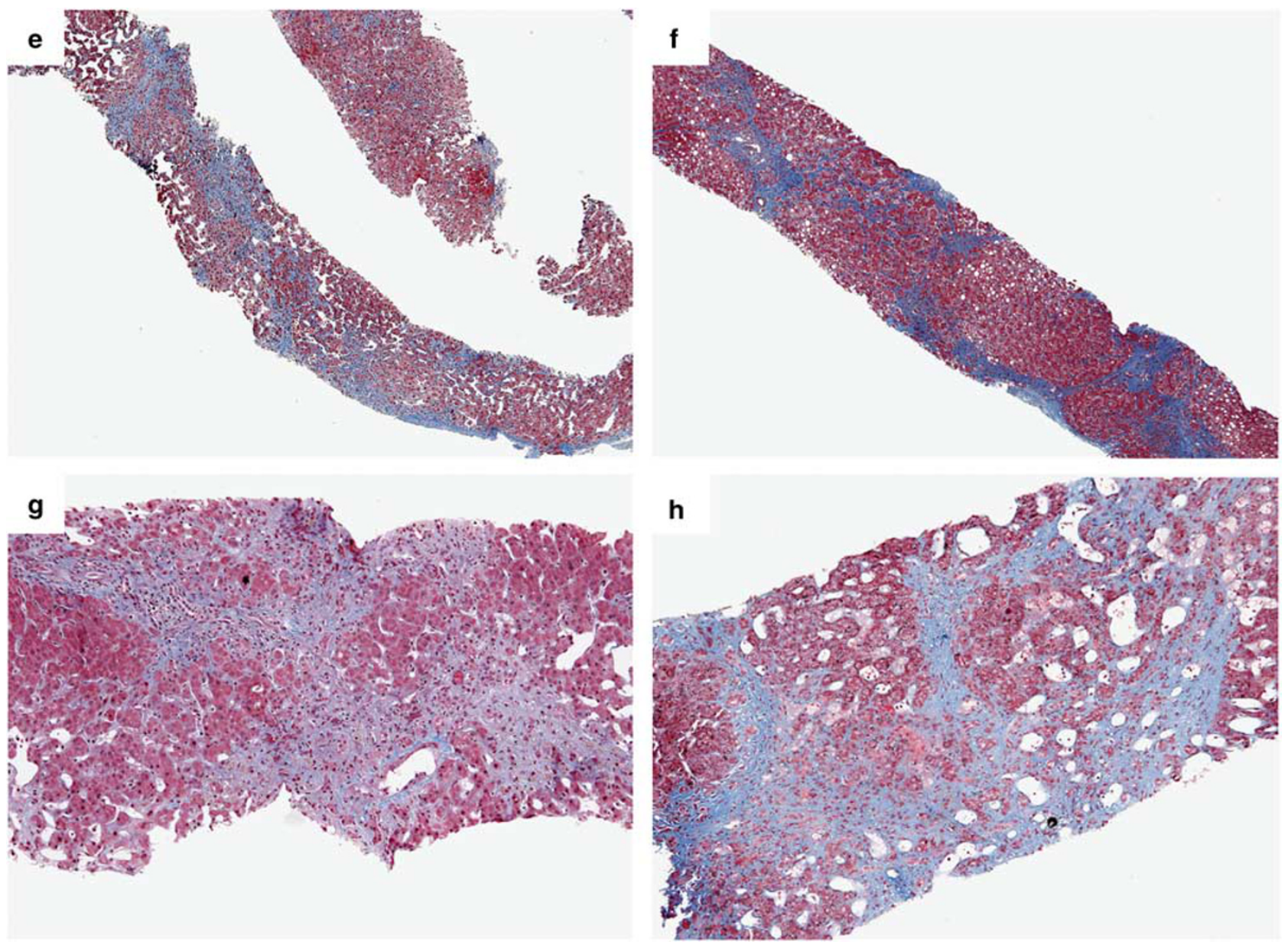

Figure 2 (Continued)

Our series of explants also permitted some assessment of the role of vascular thrombosis in cardiac hepatopathy. Wanless et al. ${ }^{19}$ noted marked variation in fibrosis in cardiac hepatopathy and suggested that hepatic venous thrombosis might contribute to the fibrosis and explain the patchy distribution of fibrosis. We found evidence of vascular remodeling on EVG stains in two of our explants, suggesting remote thrombosis. While the marked variation in fibrosis seen in these livers may be explained by thrombotic events, we could not be certain that the thrombosis was a cause of the congestion, stasis and fibrosis rather than an effect. Our evaluation did not disclose any other candidate explanation for the highly variable distribution of fibrosis.

We also noted ductular or biliary metaplasia of liver cells adjacent to central veins and centrizonal arterioles in both the explants and in the core biopsies. This change has previously been reported by Krings et al. ${ }^{21}$ Similar to these authors, we found that this lesion can be a cause of confusion such that the biliary-type epithelium may suggest a portal zone and the ductular proliferation may further suggest a large duct obstruction or sepsis. In fact, we found that most portal tracts lacked a significant ductular reaction. This phenomenon was more difficult to recognize in a core biopsy than in an explant.

The group of patients who underwent heart transplant without concurrent liver transplant experienced an overall low rate of perioperative mortality but a higher rate of 1 year mortality. Pretransplant laboratory values showed that many patients had abnormal liver function parameters. Several patients showed an INR of $>2.0$, but this was often related to warfarin therapy. In the pretransplant liver biopsies, all but three cases showed some degree of sinusoidal congestion. The majority of the cases showed increased fibrosis, generally in a pericentral and/or pericellular distribution. Out of 13 cases that showed bridging fibrosis on liver biopsy, one died in the immediate post-operative period (first month post-transplant) and an additional 4 died during the first year post transplant (3 of these 4 experienced prolonged liver failure, which we defined as persistent ascites and/or encephalopathy). All of the remaining eight patients without bridging fibrosis also survived the immediate post-operative period and all but one survived the first year. A statistically significant difference was not found in 1 year survival between the patients with and without 
Table 4 Laboratory values for patients 6 months post-transplant

\begin{tabular}{|c|c|c|c|c|c|c|c|c|}
\hline $\begin{array}{l}\text { Patient } \\
\text { number }\end{array}$ & $\begin{array}{l}\text { Age/ } \\
\text { Gender }\end{array}$ & Heart disease & $\begin{array}{c}A P \\
(I U / I)\end{array}$ & $\begin{array}{c}\text { Total } \\
\text { bilirubin }(\mathrm{mg} / \mathrm{dl})\end{array}$ & $\begin{array}{l}A S T \\
(I U / I)\end{array}$ & $A L T(I U / I)$ & $\underset{(g / d l)}{\text { Albumin }}$ & $\begin{array}{c}\text { Total } \\
\text { protein }(\mathrm{g} / \mathrm{dl})\end{array}$ \\
\hline 1 & $53 / \mathrm{F}$ & Ischemic & 129 & 1.2 & 22 & 19 & 3.5 & 6.1 \\
\hline 2 & $48 / \mathrm{M}$ & Arrhythmogenic RV cardiomyopathy & 106 & 0.9 & 39 & 46 & 4.1 & 6.6 \\
\hline 3 & $53 / \mathrm{M}$ & Dilated cardiomyopathy & 204 & 0.5 & 19 & 11 & 3.8 & 7 \\
\hline 4 & $61 / \mathrm{M}$ & Ischemic & 98 & 0.8 & 26 & 36 & 3.7 & 7 \\
\hline 5 & $66 / \mathrm{M}$ & Dilated cardiomyopathy & 82 & 0.9 & 29 & 27 & 4.3 & 6.9 \\
\hline 6 & $50 / \mathrm{M}$ & Hypertrophic cardiomyopathy & 324 & 1.8 & 354 & 135 & 2.1 & 3.9 \\
\hline 7 & $63 / \mathrm{M}$ & Ischemic & 89 & 0.5 & 25 & 41 & 4.2 & 7.5 \\
\hline 8 & $60 / \mathrm{M}$ & Amyloidosis & \multicolumn{6}{|c|}{ Deceased at 70 days } \\
\hline 9 & $40 / \mathrm{M}$ & Dilated cardiomyopathy & 153 & 0.5 & 41 & 70 & 3.1 & 9.2 \\
\hline 10 & $55 / \mathrm{F}$ & Primary pulmonary hypertension & 220 & 0.5 & 21 & 34 & 3.4 & 7.1 \\
\hline 11 & $43 / \mathrm{F}$ & Adriamycin cardiomyopathy & 560 & 1.4 & 25 & 57 & 2.3 & 6 \\
\hline 12 & $42 / \mathrm{F}$ & Congenital heart disease & 374 & 1 & 23 & 35 & 2.5 & 6.7 \\
\hline 13 & $57 / \mathrm{F}$ & Dilated cardiomyopathy & 113 & 0.5 & 28 & 39 & 4.4 & 7.3 \\
\hline 14 & $36 / F$ & Congenital heart disease & 493 & 0.5 & 106 & 163 & 3.4 & 9.1 \\
\hline 15 & $36 / \mathrm{F}$ & Restrictive cardiomyopathy & 161 & 0.6 & 24 & 36 & 3.4 & 6.8 \\
\hline 16 & $36 / \mathrm{M}$ & Congenital heart disease & \multicolumn{6}{|c|}{ Deceased at 35 davs } \\
\hline 17 & $37 / \mathrm{F}$ & Congenital heart disease & 110 & 0.8 & 25 & 77 & 4 & 6.4 \\
\hline 18 & $15 / \mathrm{M}$ & Congenital heart disease & \multicolumn{6}{|c|}{ Deceased at 19 days } \\
\hline 19 & $51 / \mathrm{M}$ & Primary pulmonary hypertension & 420 & 1.9 & 57 & 123 & 3.4 & 7.3 \\
\hline 20 & $49 / \mathrm{M}$ & Congenital heart disease & 350 & 0.6 & 23 & 9 & 2.4 & 5.5 \\
\hline 21 & $34 / \mathrm{M}$ & Restrictive cardiomyopathy & \multicolumn{6}{|c|}{ Deceased at 3 months } \\
\hline
\end{tabular}

Abbreviations: AST, aspartate aminotransferase; ALT, alanine aminotransferase; AP, alkaline phosphatase; RV, right ventricle.

Table 5 Laboratory values for patients 1 year post-transplant

\begin{tabular}{|c|c|c|c|c|c|c|c|c|}
\hline $\begin{array}{l}\text { Patient } \\
\text { number }\end{array}$ & $\begin{array}{l}\text { Age/ } \\
\text { Gender }\end{array}$ & Heart disease & $A P(I U / I)$ & $\begin{array}{c}\text { Total } \\
\text { bilirubin }(\mathrm{mg} / \mathrm{dl})\end{array}$ & $\begin{array}{c}A S T \\
(I U / l)\end{array}$ & $\begin{array}{l}A L T \\
(I U / l)\end{array}$ & Albumin $(g / d l)$ & $\begin{array}{c}\text { Total } \\
\text { protein }(\mathrm{g} / \mathrm{dl})\end{array}$ \\
\hline 1 & $53 / \mathrm{F}$ & Ischemic & 267 & 1.0 & 21 & 18 & 3.9 & 7.8 \\
\hline 2 & $48 / \mathrm{M}$ & Arrhythmogenic RV cardiomyopathy & 75 & 0.9 & 38 & 51 & 4.2 & 6.5 \\
\hline 3 & $53 / \mathrm{M}$ & Dilated cardiomyopathy & 194 & 0.9 & 18 & 18 & 4 & 6.9 \\
\hline 4 & $61 / \mathrm{M}$ & Ischemic & 85 & 0.8 & 50 & 97 & 3.9 & 7.4 \\
\hline 5 & $66 / \mathrm{M}$ & Dilated cardiomyopathy & 100 & 0.8 & 28 & 23 & 4.6 & 7.3 \\
\hline 6 & $50 / \mathrm{M}$ & Hypertrophic cardiomyopathy & \multicolumn{6}{|c|}{ Deceased at 6 months } \\
\hline 7 & $63 / \mathrm{M}$ & Ischemic & 138 & 0.5 & 28 & 42 & 4.4 & 7.5 \\
\hline 8 & $60 / \mathrm{M}$ & Amyloidosis & \multicolumn{6}{|c|}{ Deceased at 70 days } \\
\hline 9 & $40 / \mathrm{M}$ & Dilated cardiomyopathy & 171 & 0.5 & 18 & 41 & 4 & 7.6 \\
\hline 10 & $55 / \mathrm{F}$ & Primary pulmonary hypertension & 460 & 0.5 & 22 & 36 & 3.8 & 7.9 \\
\hline 11 & $43 / \mathrm{F}$ & Adriamycin cardiomyopathy & \multicolumn{6}{|c|}{ No data available } \\
\hline 12 & $42 / \mathrm{F}$ & Congenital heart disease & 643 & 0.5 & 45 & 69 & 3.3 & 7.8 \\
\hline 13 & $57 / F$ & Dilated cardiomyopathy & 186 & 0.5 & 30 & 41 & 4.2 & 7.4 \\
\hline 14 & $36 / \mathrm{F}$ & Congenital heart disease & 159 & 0.5 & 39 & 49 & 4 & 7.8 \\
\hline 15 & $36 / \mathrm{F}$ & Restrictive cardiomyopathy & 183 & 0.5 & 24 & 41 & 3.7 & 6.8 \\
\hline 16 & $36 / \mathrm{M}$ & Congenital heart disease & \multicolumn{6}{|c|}{ Deceased at 35 days } \\
\hline 17 & $37 / \mathrm{F}$ & Congenital heart disease & 115 & 0.9 & 32 & 68 & 4.2 & 6.7 \\
\hline 18 & $15 / \mathrm{M}$ & Congenital heart disease & \multirow{2}{*}{\multicolumn{6}{|c|}{$\begin{array}{c}\text { Deceased at } 19 \text { days } \\
\text { No data available }\end{array}$}} \\
\hline 19 & $51 / \mathrm{M}$ & Primary pulmonary hypertension & & & & & & \\
\hline 20 & $49 / \mathrm{M}$ & Congenital heart disease & 284 & 3 & 61 & 32 & $<1.0$ & 2.6 \\
\hline 21 & $34 / \mathrm{M}$ & Restrictive cardiomyopathy & \multicolumn{6}{|c|}{ Deceased at 3 months } \\
\hline
\end{tabular}

Abbreviations: AST, aspartate aminotransferase; ALT, alanine aminotransferase; AP, alkaline phosphatase; RV, right ventricle.

bridging fibrosis, although this may be related to the small sample size. The findings suggest that fibrosis by itself on a biopsy should not preclude a patient's candidacy for heart transplantation. However, three patients developed clinically significant chronic liver disease within the first year post-transplantation, suggesting that the finding of bridging fibrosis on a liver biopsy, even in carefully selected patients, may be a marker of higher risk. Our data add to the existing evidence in the literature that patients with cardiac hepatopathy may, if carefully selected after considering additional clinical and laboratory parameters, be appropriate candidates for cardiac transplantation. . $^{811,13,14}$

Our findings are in agreement with studies that have shown that after successful cardiac 
transplantation, patients may show improvement of liver disease in terms of both laboratory values and histologic findings. ${ }^{11,13,14}$ Our patients all showed marked improvement in liver enzymes and bilirubin levels at 6 months and further improvement at 1 year post-transplant with normal levels observed in most patients. One patient (patient \#3) showed decreased fibrosis on a follow-up liver biopsy 6 years after heart transplant, providing some evidence that fibrosis in the context of chronic passive congestion may be, to some degree, reversible; however, these findings may also be subject to the limitations of biopsy sampling and the possibility of patchy fibrosis. While we do not have sufficient data to suggest that fibrosis necessarily resolves post-transplant, our findings clearly show that liver injury is not progressive once normal cardiac function is restored.

In a previous study by Chokshi et al., ${ }^{11}$ patients undergoing heart transplant with higher preoperative MELD scores were found to have worse outcomes in the immediate post-transplant period in addition to reduced long-term survival over 10 years. Additionally, recent studies have suggested that MELD scores predict survival in patients undergoing orthotopic heart transplant. ${ }^{24}$ Our data show that pre-transplant MELD scores were not significantly associated with the presence of fibrosis on biopsy. Furthermore, our patients who died or developed liver disease post-transplant did not have higher MELD scores than our patients who did well; the patients with pre-operative MELD scores above 20 were either still alive at follow-up or died of causes unrelated to liver failure. However, our cohort was highly selected, and patients with elevated MELD scores or clinical evidence of cirrhosis may not have undergone further invasive evaluation. Additionally, patients with significant fibrosis on biopsy may have been judged eligible for transplant if their MELD score was low, which may be a source of selection bias in our cohort.

While the uneven distribution of fibrosis in livers with chronic passive congestion limits the clinical significance of findings on liver biopsy, biopsies continue to be performed to stage the fibrosis. The orderly progression of changes observed suggests a possible staging scheme. We propose that liver injury due to chronic passive congestion be staged as follows: stage 0-no fibrosis; stage I-any degree of pericellular fibrosis short of central-central or central-portal bridging; stage II-bridging fibrosis, either central-central or central-portal; and stage IIIregenerative nodules of hepatocytes (Table 3). Despite the fact that the fibrotic changes secondary to chronic passive congestion seem to follow a stepwise pattern, we believe that livers showing the presence of regenerative nodules on biopsy should not be categorized as cirrhotic. A diagnosis of cirrhosis requires that the entire liver be affected by fibrosis and nodular regeneration, and thus should only be diagnosed on an explant specimen. While it must be recognized that a biopsy is susceptible to sampling error, particularly given the potentially patchy nature of fibrosis in the context of chronic passive congestion, this staging scheme may provide some insight into the degree of chronic damage present in at least the biopsied portion of the liver. Since the staging scheme we propose depends on a dichotomous assessment for fibrosis, bridging, and regenerative nodules, we anticipate that it may be relatively easily employed and reproducible.

Our study has several limitations. Judging whether patients suffered bleeding or infectious complications as a result of their liver disease proved problematic. While chronic liver disease has been reported to predispose to both infection and bleeding, both also commonly occur in patients undergoing major surgery followed by intensive immunosuppression and the threshold at which post-operative bleeding becomes excessive is not well established. Five patients experienced serious infectious complications at some point in their post-transplant course while post-operative bleeding was noted in six. We were unable to determine whether these complications were directly related to or exacerbated by the patients' underlying liver disease.

Another limitation is that this is a retrospective study with inherent selection bias. Both patient populations (those undergoing combined heart and liver versus heart only) were carefully selected to undergo each procedure on the basis of their perceived risk of peri-operative and post-transplant survival. Because we lack robust published data in the literature on risk stratification in cardiac-related liver disease and selection guidelines for multi-organ transplant, the patients were selected for each procedure on the basis of various factors, including chronicity and severity of heart failure, the presence or absence of stigmata of chronic liver disease, abdominal imaging results, liver function tests, and markers of synthetic function, age, the presence of other co-morbidities, and interpretation of liver biopsy results. Because rigid criteria were not used, it is often not apparent in retrospect which factors led the clinical team to recommend a combined heart-liver transplant versus isolated heart transplant. In fact, the MELD scores and biopsy findings of patients who underwent combined heart-liver transplant are not clearly worse than many patients who underwent isolated heart transplant. Furthermore, the group of patients who undergo liver biopsy represent a specific population for whom chronic liver damage is suspected but the extent of liver injury is unclear based on clinical parameters alone. Therefore, some amount of selection bias exists as patients without clinical, laboratory, or radiographic sequelae of chronic liver disease, and patients with clear cut cirrhosis based on imaging studies will likely not undergo a biopsy. Finally, another major limitation of our study is the small sample size. Further study of additional patients will clearly provide insight into the implications of biopsy and other clinical findings in this population. 
In summary, our findings suggest that the liver biopsy results alone should not be used to exclude patients from consideration of heart transplantation but may help to further risk stratify patients with poor hepatic reserve who may develop complications of chronic liver disease early posttransplantation. Additional studies, however, are still needed to further understand the mechanisms for the histologic changes seen in livers of patients with heart failure, to identify patients in whom liver disease is expected to recover after normalization of cardiac hemodynamics, and to aid in more accurately determining operative risk and post-transplant survival in patients who are undergoing evaluation for heart transplantation.

\section{Disclosure/conflict of interest}

The authors declare no conflict of interest.

\section{References}

1 Giallourakis CC, Rosenberg PM, Friedman LS. The liver in heart failure. Clin Liver Dis 2002;6:947-967.

2 Samsky MD, Patel CB, DeWald TA et al. Cardiohepatic interactions in heart failure: an overview and clinical implications. J Am Coll Cardiol 2013;61: 2397-2405.

3 Garrison RN, Cryer HM, Howard DA et al. Clarification of risk factors for abdominal operations in patients with hepatic cirrhosis. Ann Surg 1984;199:648-655.

4 Mansour A, Watson W, Shayani V et al. Abdominal operations in patients with cirrhosis: still a major surgical challenge. Surgery 1997;122:730-735.

5 Neeff H, Mariaskin D, Spangenberg HC et al. Perioperative mortality after non-hepatic general surgery in patients with liver cirrhosis: an analysis of 138 operations in the 2000s using Child and MELD scores. J Gastrointest Surg 2011;15:1-11.

6 Filsoufi F, Salzberg SP, Rahmanian PB et al. Early and late outcome of cardiac surgery in patients with liver cirrhosis. Liver Transpl 2007;13:990-995.

7 Hsu RB. Heart transplantation in patients with endstage heart failure and cardiac ascites. Circ J 2007;71: 1744-1748.

8 Hsu RB, Chang CI, Lin FY et al. Heart transplantation in patients with liver cirrhosis. Eur J Cardiothorac Surg 2008;34:307-312.

9 Suman A, Barnes DS, Zein NN et al. Predicting outcome after cardiac surgery in patients with cirrhosis: a comparison of Child-Pugh and MELD scores. Clin Gastroenterol Hepatol 2004;2:719-723.

10 Kramer MR, Marshall SE, Tiroke A et al. Clinical significance of hyperbilirubinemia in patients with pulmonary hypertension undergoing heart-lung transplantation. J Heart Lung Transplant 1991;10:317-321.
11 Chokshi A, Cheema FH, Schaefle KJ et al. Hepatic dysfunction and survival after orthotopic heart transplantation: application of the MELD scoring system for outcome prediction. J Heart Lung Transplant 2012;31: 591-600.

12 Singh TP, Almond CS, Semigran MJ et al. Risk prediction for early in-hospital mortality following heart transplantation in the United States. Circ Heart Fail 2012;5:259-266.

13 Crespo-Leiro MG, Robles O, Paniagua MJ et al. Reversal of cardiac cirrhosis following orthotopic heart transplantation. Am J Transplant 2008;8:1336-1339.

14 Dichtl W, Vogel W, Dunst KM et al. Cardiac hepatopathy before and after heart transplantation. Transpl Int 2005;18:697-702.

15 Simpson KE, Esmaeeli A, Khanna G et al. Liver cirrhosis in Fontan patients does not affect 1-year post-heart transplant mortality or markers of liver function. J Heart Lung Transplant 2014;33:170-177.

16 Costanzo MR, Augustine S, Bourge R et al. Selection and treatment of candidates for heart transplantation. A statement for health professionals from the Committee on Heart Failure and Cardiac Transplantation of the Council on Clinical Cardiology, American Heart Association. Circulation 1995;92:3593-3612.

17 Gelow JM, Desai AS, Hochberg CP et al. Clinical predictors of hepatic fibrosis in chronic advanced heart failure. Circ Heart Fail 2010;3:59-64.

18 Cannon RM, Hughes MG, Jones CM et al. A review of the United States experience with combined heart-liver transplantation. Transpl Int 2012;25:1223-1228.

19 Wanless IR, Liu JJ, Butany J. Role of thrombosis in the pathogenesis of congestive hepatic fibrosis (cardiac cirrhosis). Hepatology 1995;21:1232-1237.

20 Boland EW, Willius FA. Changes in the liver produced by chronic passive congestion: with special reference to the problem of cardiac cirrhosis. Arch Intern Med 1938;62:723-739.

21 Krings G, Can B, Ferrell L. Aberrant centrizonal features in chronic hepatic venous outflow obstruction: centrilobular mimicry of portal-based disease. Am J Surg Pathol 2014;38:205-214.

22 Kendall TJ, Stedman B, Hacking N et al. Hepatic fibrosis and cirrhosis in the Fontan circulation: a detailed morphological study. J Clin Pathol 2008;61: 504-508.

23 Lefkowitch JH, Mendez L. Morphologic features of hepatic injury in cardiac disease and shock. J Hepatol 1986;2:313-327.

24 Kim MS, Kato TS, Farr M et al. Hepatic dysfunction in ambulatory patients with heart failure: application of the MELD scoring system for outcome prediction. J Am Coll Cardiol 2013;61:2253-2261.

25 Arcidi JM Jr., Moore GW, Hutchins GM. Hepatic morphology in cardiac dysfunction: a clinicopathologic study of 1000 subjects at autopsy. Am J Pathol 1981; 104:159-166.

26 Sherlock S. The liver in heart failure; relation of anatomical, functional, and circulatory changes. $\mathrm{Br}$ Heart J 1951;13:273-293. 